\title{
Nanomedicine in Action: An Overview of Cancer Nanomedicine on the Market and in Clinical Trials
}

\author{
Ruibing Wang, Paul S. Billone, and Wayne M. Mullett \\ Nordion Inc., 447 March Road, Ottawa, ON, Canada K2K 1X8 \\ Correspondence should be addressed to Ruibing Wang; ruibing.wang@nordion.com
}

Received 22 October 2012; Accepted 9 November 2012

Academic Editor: Haiyan Li

Copyright (C) 2013 Ruibing Wang et al. This is an open access article distributed under the Creative Commons Attribution License, which permits unrestricted use, distribution, and reproduction in any medium, provided the original work is properly cited.

Nanomedicine, defined as the application of nanotechnology in the medical field, has the potential to significantly change the course of diagnostics and treatment of life-threatening diseases, such as cancer. In comparison with traditional cancer diagnostics and therapy, cancer nanomedicine provides sensitive cancer detection and/or enhances treatment efficacy with significantly minimized adverse effects associated with standard therapeutics. Cancer nanomedicine has been increasingly applied in areas including nanodrug delivery systems, nanopharmaceuticals, and nanoanalytical contrast reagents in laboratory and animal model research. In recent years, the successful introduction of several novel nanomedicine products into clinical trials and even onto the commercial market has shown successful outcomes of fundamental research into clinics. This paper is intended to examine several nanomedicines for cancer therapeutics and/or diagnostics-related applications, to analyze the trend of nanomedicine development, future opportunities, and challenges of this fast-growing area.

\section{Introduction}

According to National Nanotechnology Initiative (NNI, a multiagency US government program initiated in 2001), nanotechnology is broadly defined as the science and engineering involved in the design, synthesis, characterization, and application of materials and devices with at least one of the dimensions on the nanoscale (typically 1-100 nanometers) [1]. Since its inception several decades ago, nanotechnology has drawn increasing attention from both the academic and industrial sectors for applications not only in materials science and engineering, such as light-emitting devices and solar cells, but also in the biotechnology and medical fields including disease diagnostics, prevention, and treatment. Accordingly, the level of interest in nanotechnology shown by both academic and industrial investigators has led to the increased development of novel nanotechnology platforms for medical applications, sharp increases in government funding, and venture capital investment [2]. Nanomedicine as a newly created subterm, refers to the application of engineered nanomaterials to the medical field.

Nanotechnology offers many potential benefits to medical applications including the early detection of cancers and cancer treatment, passive and active disease targeting, increased biocompatibility, and multifunctionality encompassing both imaging and therapeutic capabilities, allowing for simultaneous disease treatment and monitoring [3]. The major advantages of nanomaterials in medical applications include the following. (1) They are on the same size scale with biomolecules such as receptors, antibodies, and nucleic acids. Nanomaterials can also be functionalized with biomolecules, enabling them to target specific organelles within certain tissues or even the entire cells for localization in the targeted area. (2) Nanostructures can often overcome solubility and stability issues through surface modification/wrappings or additional formulation. (3) Nanostructures have novel physical properties, such as optical properties from quantum dots, which can be utilized for bioimaging. (4) Due to the nanosize, they are normally composed of thousands of atoms with a high surface area so that a higher therapeutic payload (e.g., radioactive isotopes or chemotherapy drugs) can be carried to or encapsulated in the nanostructure. Once delivered and recognized by a receptor, the high-dose therapeutic load can cause more devastating damage to cancer cells at the targeted site. (5) Nanoparticle (NP) formulations, through passive or active targeting, can often release therapeutic payloads at 
TABLE 1: Nanomedicines approved by one or more regulatory bodies.

\begin{tabular}{|c|c|c|c|c|}
\hline Product & Nanoplatform/agent & Indication & Status & Company \\
\hline Doxil & $\begin{array}{l}\text { PEGylated } \\
\text { liposome/doxorubicin } \\
\text { hydrochloride }\end{array}$ & Ovarian cancer & $\begin{array}{l}\text { Approved 11/17/1995 } \\
\text { FDA50718 }\end{array}$ & $\begin{array}{l}\text { Ortho Biotech } \\
\text { (acquired by JNJ) }\end{array}$ \\
\hline Myocet & $\begin{array}{l}\text { Non-PEGylated liposomal } \\
\text { doxorubicin nanomedicine }\end{array}$ & Metastatic breast cancer & $\begin{array}{l}\text { Approved in Europe and } \\
\text { Canada, in combination } \\
\text { with cyclophosphamide }\end{array}$ & $\begin{array}{l}\text { Sopherion Therapeutics, } \\
\text { LLC in North America and } \\
\text { Cephalon, Inc. in Europe }\end{array}$ \\
\hline DaunoXome & $\begin{array}{l}\text { Lipid encapsulation of } \\
\text { daunorubicin }\end{array}$ & $\begin{array}{l}\text { First-line treatment for } \\
\text { patients with advanced } \\
\text { HIV-associated Kaposi's } \\
\text { sarcoma }\end{array}$ & Approved in the USA & Galen Ltd. \\
\hline ThermoDox & $\begin{array}{l}\text { Heat-activated liposomal } \\
\text { encapsulation of } \\
\text { doxorubicin }\end{array}$ & $\begin{array}{l}\text { Breast cancer, primary liver } \\
\text { cancer }\end{array}$ & $\begin{array}{l}\text { Received Fast Track } \\
\text { Designation, approval } \\
\text { expected by } 2013\end{array}$ & Celsion \\
\hline Abraxane & $\begin{array}{l}\text { Nanoparticulate } \\
\text { albumin/paclitaxel }\end{array}$ & Various cancers & $\begin{array}{l}\text { Approved 1/7/2005 } \\
\text { FDA21660 }\end{array}$ & Celgene \\
\hline Rexin-G & $\begin{array}{l}\text { Targeting protein tagged } \\
\text { phospholipid/microRNA- } \\
122\end{array}$ & $\begin{array}{l}\text { Sarcoma, osteosarcoma, } \\
\text { pancreatic cancer, and } \\
\text { other solid tumor }\end{array}$ & $\begin{array}{l}\text { Fully approved in } \\
\text { Philippine } \\
\text { Phase II/III (Fast Track } \\
\text { Designation, Orphan Drug } \\
\text { Status Acquired) in USA }\end{array}$ & $\begin{array}{l}\text { Epeius Biotechnologies } \\
\text { Corp. }\end{array}$ \\
\hline Oncaspar & PEGylated asparaginase & $\begin{array}{l}\text { Acute lymphoblastic } \\
\text { leukemia }\end{array}$ & Approved 24/06/2006 & $\begin{array}{l}\text { Enzon Pharmaceuticals, } \\
\text { Inc. }\end{array}$ \\
\hline Resovist & $\begin{array}{l}\text { Iron oxide nanoparticles } \\
\text { coated with carboxydextran }\end{array}$ & Liver/spleen lesion imaging & $\begin{array}{l}\text { In 2001, approved for the } \\
\text { European market }\end{array}$ & Bayer Schering Pharma AG \\
\hline Feridex & $\begin{array}{l}\text { Iron oxide nanoparticles } \\
\text { coated with dextran }\end{array}$ & Liver/spleen lesion imaging & $\begin{array}{l}\text { Approved by US-FDA in } \\
1996\end{array}$ & Berlex Laboratories \\
\hline Endorem & $\begin{array}{l}\text { Iron oxide nanoparticles } \\
\text { coated with dextran }\end{array}$ & Liver/spleen lesion imaging & Approved in Europe & Guerbet \\
\hline
\end{tabular}

cancer sites, thus significantly reducing nonspecific toxicity [3].

However, challenges always exist together with opportunities. First, nanomedicine may pose a myriad of full characterization challenges. Second, concerns about safety and manufacturability should not be ignored. For instance, each nanomedicine's batch-to-batch consistency needs to be verified with effective quality control methods [4]. In addition, nanomedicine may be ineffective if the therapeutic moiety of a particle disassociates from the nanoparticle platform upon administration or during circulation in the bloodstream or is degraded inside the particle. Pretargeting release of a therapeutic payload (e.g., a chemotherapeutic) from the nanoplatform may result in an acute toxicity independent of the pharmacokinetics of the nanoparticle platform itself $[3,5]$. Clinical translation of NPs requires a complete understanding of particle size, composition, formulation, internal and external structure, chemical reactivity and stability, and their relationship with the human body, namely, biodistribution, toxicity, and biocompatibility [6].

Even with these and other challenges, a few firstgeneration nanomedicines have already obtained recognition in the clinical cancer research community as effective tools. For instance, Doxil (a liposomal formulation of doxorubicin) was approved by the FDA in the mid-1990s and demonstrated a decreased cardiotoxicity compared with free doxorubicin
TABLE 2: Half-life of ASNase (native versus PEGylated) [50].

\begin{tabular}{lcc}
\hline Product & Manufacturer & $T_{1 / 2}$ \\
\hline E. coli native ASNase (Elspar) & Merck \& Co. Inc. & 1.15 days \\
PEG-ASNase (Oncaspar) & Enzon Pharmaceuticals & 5.85 days \\
\hline
\end{tabular}

[7]. Another prime example of the budding success of nanomedicine is Abraxane, a nanoscale albumin-bound form of paclitaxel, approved by FDA in 2005 [8]. This nanoparticle has a size around $100 \mathrm{~nm}$ and solubilizes a poorly soluble drug (paclitaxel) so that the notable side effects of paclitaxel are significantly decreased. Tables 1 and 3 list nanomedicine, constructs currently on the market and in clinical trials, respectively. It is noteworthy from the list that most of the FDA approved marketed nanomedicines, including Doxil, Abraxane, ThermoDox, and Rexin-G, are organic-based formulations, presumably due to better biocompatibility and the low (or no) toxicity of the organic platforms. However, inorganic materials-based medical devices and nanomedicines, such as Aurimune (colloidal gold platform based) and AuroLase (gold-coated silica nanoparticle platform based), have recently gained FDA recognition and are in various phases of clinical trials $[9,10]$. The majority of the agents are therapeutics, where market sizes are larger and potentially more profitable than diagnostics, given equal or similar 


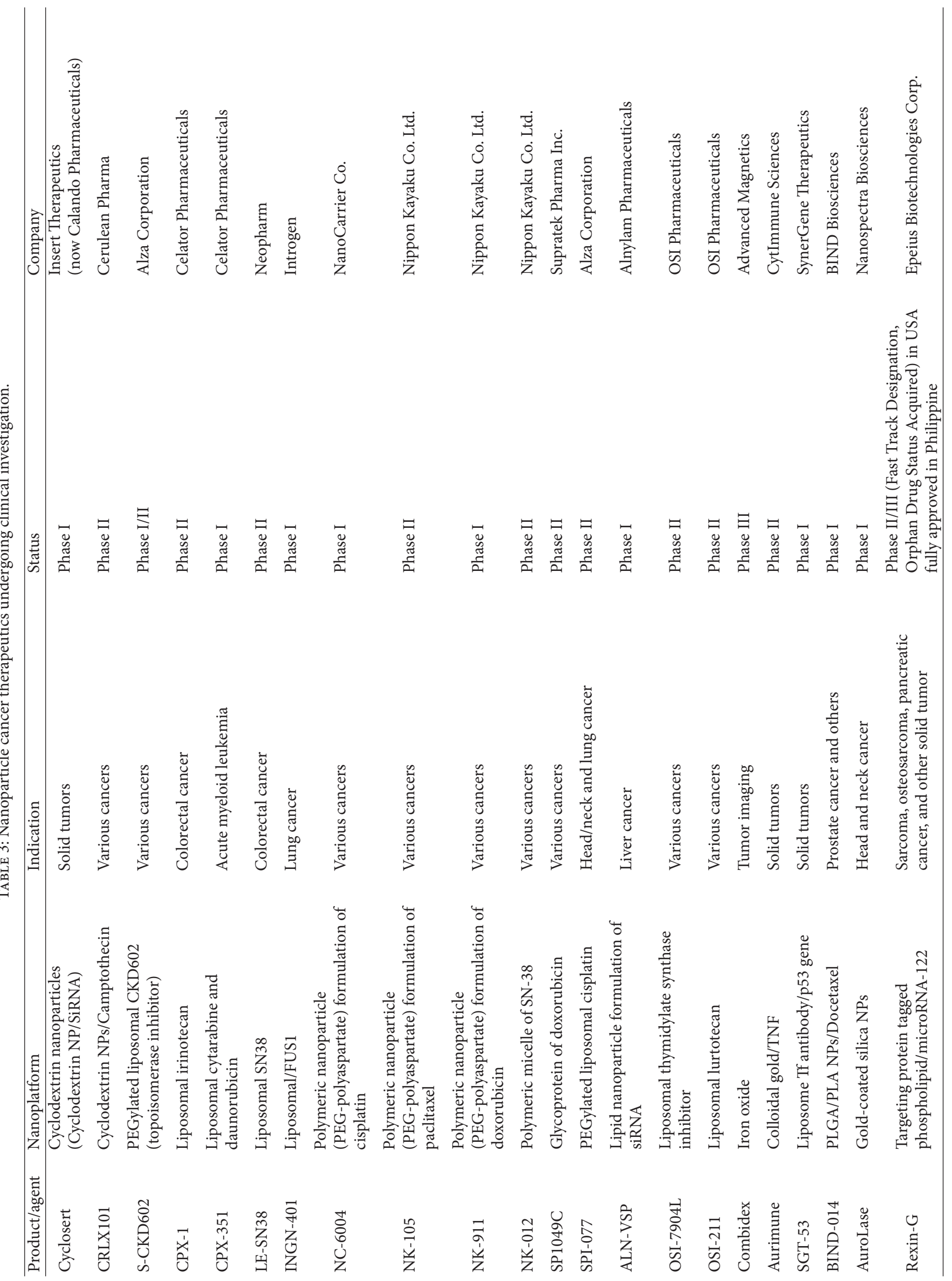




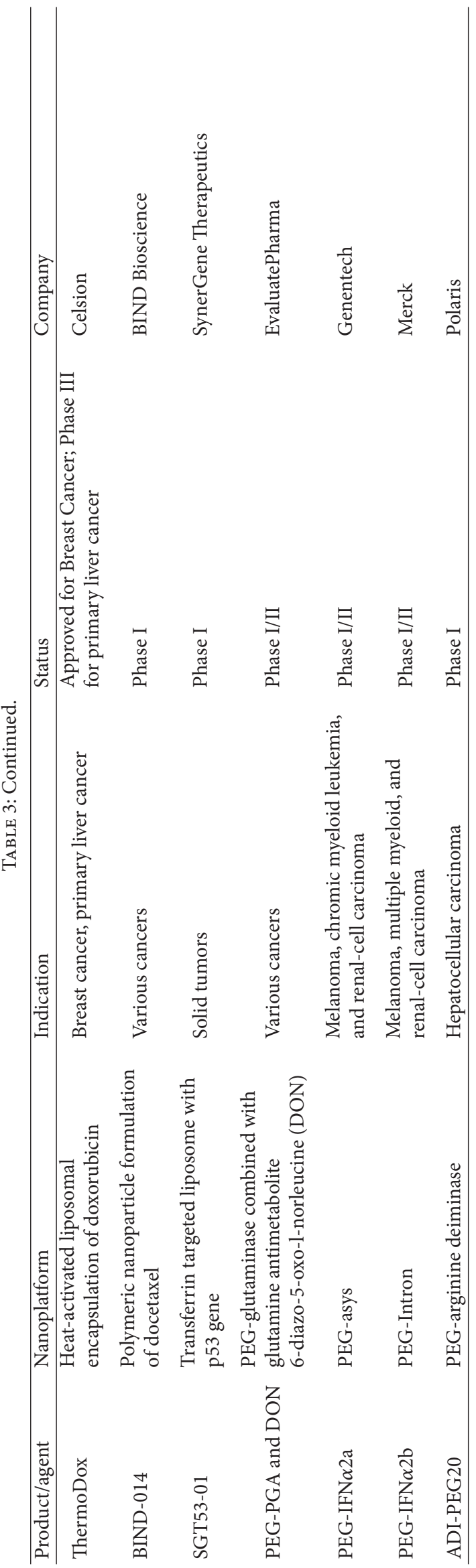


development costs associated with a regulatory approval for either type [6].

The following sections will discuss nanomedicines that are on the market (approved from one or more regulatory authorities for commercial use) and nanomedicines that are still in clinical trials, as listed in Tables 1 and 3, respectively.

\section{Cancer Nanomedicine on the Market}

2.1. Liposomal Anthracyclines. There are several nanoparticle technologies based on the liposomal encapsulation of anthracyclines, which are known to be potent cytotoxic agents for various types of cancers. Three of the most widely known and marketed technologies are Doxil (also marketed in Canada and Europe as Caelyx, Janssen Biotech, Inc.), Myocet (marketed by Sopherion Therapeutics, LLC in North America and Cephalon, Inc. in Europe), and DaunoXome (Galen Ltd.).

2.1.1. Doxil. Doxil was initially approved by the FDA in 1995 as a treatment for AIDS-related Kaposi's sarcoma. It is considered the first approved "nanodrug" and has been successful since its market introduction, with over $\$ 600$ million USD in annual sales across all of its markets [11].

Doxil liposomes consist of a single lipid bilayer membrane composed of hydrogenated soy phosphatidylcholine (HSPC) and cholesterol with doxorubicin encapsulated in the internal compartment [12]. The mean size of the vesicles is in the range of $80-90 \mathrm{~nm}$ [13] and because doxorubicin is a small-molecule therapeutic, each vesicle can hold a payload of up to 15,000 molecules [12]. In order to mitigate the stability and early release issues that traditional liposomal encapsulations of doxorubicin have [14], $2000 \mathrm{Da}$ segments of poly(ethylene glycol) (PEG) are grafted to the liposome surface. In addition to providing stability to the particles, the PEG functionalization makes the particles nearly invisible to the reticuloendothelial system (RES), earning them the distinction of stealth liposomes $[12,13]$.

Doxil vesicles rely on a passive targeting mechanism in order to accumulate at tumor sites [13]. While not specific to the Doxil formulation (as evident from the other technologies discussed below), the primary mechanism for accumulation and distribution throughout tumor sites is believed to be due to the combination of long circulation time (half-life of 2-3 days for clearance) [12] and the microvascularity of tumors, along with the enhanced permeability and retention (EPR) effect $[15,16]$. The volume of the distribution of Doxil is only slightly larger than the plasma volume itself, indicating that there is a very little uptake of the liposomes by healthy tissue. Consequently, many studies have indicated that Doxil efficacy is substantially higher than that of free doxorubicin on a mg-to-mg scale [13], likely due to the liposomal targeting mechanism discussed above. However there is still no consensus on the mechanism of doxorubicin delivery itself to the cancerous cells within the tumor once the vesicles have been uptaken. Barenholz discusses two mechanisms [11] but concludes that the most likely mechanism for doxorubicin delivery is that a collapse of the ammonium sulfate gradient in the tumor interstitial fluid results in the release of the doxorubicin outside the tumor cells, after which it is taken up by the cells; this mechanism still lacks conclusive clinical evidence.

Despite its significantly longer circulation time than doxorubicin itself (which has an in vivo half-life of $\sim 5 \mathrm{~min}$ ) [12], Doxil has dramatically different, and less severe, side effects than the free drug [15]. In particular, Doxil shows a drastic decrease in the cardiotoxicity over doxorubicin, for which cardiotoxicity is the dose-limiting side effect. Early Phase I-II studies with Doxil revealed that the cardiotoxicity in solid tumor patients is insignificant [12], attributed to nearly negligible levels of the free drug in the blood stream and the minimal distribution of Doxil to the heart itself. The maximum tolerated dose (MTD) of Doxil, as defined during Phase II trials, was $50 \mathrm{mg} / \mathrm{m}^{2}$ every four weeks [17]. The two most severe side effects of Doxil are mucositis and palmar plantar erythrodysesthesia (PPE) [12]. PPE, which is a schedulelimiting and critical dose-limiting factor for multiple-course treatment regimes [12], is a toxic effect unique to Doxil (not observed with free doxorubicin) and is attributed largely to the long circulation time of the vesicles and a tendency of stealth liposomes to accumulate at the skin [18].

As of 2012, indications for Doxil include second-line treatment of AIDS-related Kaposi's sarcoma (1995), recurrent ovarian cancer (1998), metastatic breast cancer (2003), and multiple myeloma in combination with bortezomib (2007) [11].

2.1.2. Myocet. Myocet is a liposomal doxorubicin nanomedicine whose main difference from Doxil is that it lacks the PEG functionalization on the particle surface. Advantages of the formulation are mainly to do with toxicity. Without PEGylation, the circulation time is significantly shorter than observed for Doxil ( $2.5 \mathrm{hrs})$ and the liposomes are not "invisible" to the RES. Therefore, Myocet is not associated with PPE, the dose-limiting toxicity of Doxil, and shows significantly reduced incidence of mucositis when compared to Doxil [19]. However, Myocet still has a circulation time long enough to ensure the effective passive targeting of tumor sites. Despite the significantly increased circulation time of Myocet as compared to doxorubicin, efficacy is essentially the same in most clinical tests [20]. In one particular Phase III head-to-head comparison between doxorubicin and Myocet for the treatment of metastatic breast cancer, response rates and progression-free survival were identical for the two treatments [21]. However, the incidence of cardiac-related toxicity was lower with Myocet. The major dose limiting toxicity (DLT) of Myocet has been shown to be leukopenia or neutropenia [22].

The Myocet liposome is composed of a bilayer membrane of egg phosphatidylcholine and cholesterol in a $55: 45$ mole ratio. Doxorubicin is loaded into the internal aqueous core of the particle using an active loading process driven by a $\mathrm{pH}$ gradient; once the doxorubicin is loaded into the liposomes, the molecules stack into fibres that are noncovalently crosslinked with citrate [23]. Unlike Doxil, Myocet has been observed to release its doxorubicin quite rapidly. Within 24 hours, $90 \%$ of its doxorubicin content is released in vivo [19]. The increased release rate as compared to Doxil is attributed 
primarily to the lack of PEG coating, which stabilizes the membrane of the liposome and prevents the leakage of the payload [16].

Myocet is not yet approved for use in the USA (Phase III clinical trials as first-line treatment for HER2 positive metastatic breast cancer is underway) but it is marketed in Canada and Europe in combination with cyclophosphamide as a first-line treatment for metastatic breast cancer [23].

2.1.3. DaunoXome. Unlike the previous two technologies, DaunoXome is a lipid encapsulation of a different anthracycline, daunorubicin. Daunorubicin differs from the more commonly used doxorubicin in that the former lacks a hydroxyl group at the 14-position [24]. DaunoXome was originally developed using daunorubicin instead of doxorubicin due to its increased aqueous stability and its increased cytotoxicity for certain types of solid tumors [19]. The DaunoXome liposome consists of a bilayer membrane of distearoyl phosphatidylcholine and cholesterol at a $2: 1$ molar ratio. The citrate salt of daunorubicin is encapsulated within the inner aqueous core of the vesicle [25]. The particles themselves are approximately $50 \mathrm{~nm}$ in diameter [26] and demonstrate the high stability and minimal leakage of the entrapped daunorubicin [27].

Like the previously discussed doxorubicin-based technologies, DaunoXome also relies on a passive-targeting mechanism for the targeting of tumor sites. Because of the small vesicle size, net neutral charge, and incorporation of cholesterol and a lipid molecule with a high phase-transition temperature, DaunoXome is able to avoid the RES and has a circulation half-life of 2-4 hours [28]. Early trials with DaunoXome versus free daunorubicin showed higher accumulation of free daunorubicin within tumors for the DaunoXome than for the free drug. The free drug showed $9.6 \mu \mathrm{g} / \mathrm{g}$ accumulation in the tumor after $1 \mathrm{hr}$, with further decreasing levels thereafter, while DaunoXome treatment resulted in $100 \mu \mathrm{g} / \mathrm{g}$ accumulation of daunorubicin equivalents through 11 hours [24].

DaunoXome is approved in the USA as a first-line treatment for patients with advanced HIV-associated Kaposi's sarcoma [29]. There are also many active and/or recruiting clinical trials trying to prove the clinical efficacy for various forms of leukemia [30].

2.2. ThermoDox. While not currently approved in any markets, Celsion's ThermoDox is very similar to Doxil and Myocet, as discussed above; in that it is composed of doxorubicin encapsulated within the aqueous inner core of bilayer liposomes. The property that makes ThermoDox unique and has garnered it so much attention is that upon heating the liposomes to temperatures $\geq 39.5^{\circ} \mathrm{C}$, they release their payloads of doxorubicin within seconds $[31,32]$. This property enables the payload of doxorubicin to be delivered as a burst to a tumor site without the associated systemic toxicity observed with the administration of the free drug itself [33].

The ThermoDox liposome is comprised of dipalmitoylphosphatidylcholine (DPPC), monostearoylphosphatidylcholine (MSPC), and DSPE-MPEG-2000 in a 90:10:4 molar ratio [31]. DPPC, which has a gel-to-liquid crystalline phase transition temperature (Tc) of $41.5^{\circ} \mathrm{C}$, induces membrane instability in the ThermoDox liposome at temperatures around Tc. Because of its size $(\sim 100 \mathrm{~nm})$ and nature, the liposomes are rapidly identified by the RES and are concentrated in the liver [33]. Therefore, ThermoDox was identified as an ideal candidate for the treatment of hepatocellular carcinoma (HCC). In order to deliver the thermal energy required to release the ThermoDox payload of doxorubicin, radiofrequency ablation (RFA) is a convenient means as it is already widely used in the treatment of HCC. Two of the main drawbacks of RFA in the treatment of HCC tumors are the following. (1) Large tumors $(>3 \mathrm{~cm}$ ) are ineffectively treated and (2) cancerous cells at the periphery of the tumor often survive following RFA treatment and result in incidents of recurrence [33]. However, when ThermoDox was administered prior to RFA treatment in various preclinical and early-phase clinical trials, there were synergistic effects on treatment. The doxorubicin released by the ThermoDox upon RFA-induced heating increases the tumoricidal effect at the ablation margin, while RFA improves the efficacy of the doxorubicin at killing cancerous cells. The latter point has been observed through many studies and is attributed to the upregulation of doxorubicin induced at the high temperatures reached during RFA [33].

The MTD of ThermoDox was determined to be $50 \mathrm{mg} / \mathrm{m}^{2}$; the dose-limiting toxicities were grade 3 alanine aminotransferase increase and grade 4 neutropenia. There does not seem to be any abnormal liver toxicity for the treatment based on the use of ThermoDox; all of the adverse events during early phase clinical trials were consistent with the adverse event profile of free doxorubicin.

There is currently one major Phase III clinical trial ongoing for ThermoDox in conjunction with RFA for the treatment of unresectable HCC [34]. According to the information on Celsion's website (http://celsion.com/), results of the pivotal HEAT clinical trial are expected sometime in 2013. The FDA has given the HEAT trial a Fast Track Designation and it is being conducted under an FDA Special Protocol Assessment. In addition, the National Institutes of Health (NIH) has designated the study as a Priority Trial for liver cancer. The US-FDA, European Medicines Agency (EMA), and the Chinese, South Korean, and Taiwanese regulatory bodies have all confirmed that the HEAT study provides an acceptable basis for the submission of a marketing authorization application [35]. ThermoDox treatment is also being investigated for various forms of breast cancer and bone metastases [36].

2.3. Abraxane. Unlike the previously discussed liposomal technologies, Abraxane (marketed by Celgene) is a nanoparticle-based cancer treatment that utilize albumin nanoparticles as the carriers of the active agent. The active agent in Abraxane is paclitaxel, a diterpene that has dramatic chemotherapeutic effects based on its ability to stabilize microtubules causing mitotic arrest [37]. While paclitaxel has been used widely as a chemotherapeutic agent for various forms of cancer, its original formulation (known as Taxol and also including ethanol) included the solvent Cremophor, which itself caused a number of adverse reactions, including 
acute hypersensitivity [38]. Because of paclitaxel's hydrophobic nature, it needs a nonpolar carrier to make it clinically viable. Albumin is an ideal carrier as it is the natural carrier of hydrophobic molecules such as vitamins and hormones with favorable noncovalent binding interactions [39]. The nanoconjugates of albumin and paclitaxel are easily synthesized by mixing paclitaxel and human serum albumin in an aqueous solvent and passing the solution through a high pressure jet, resulting in nanoparticles in the size range of $100-200 \mathrm{~nm}$ [40]. Upon injection into biological systems, the nanoparticles rapidly dissolve into smaller $10 \mathrm{~nm}$ complexes consisting of albumin molecules with bound paclitaxel [38]. As expected, the Abraxane formulation lacks the toxicity effects attributed to the Cremophor solvent.

Abraxane has been shown to not only decrease the toxicity of traditional paclitaxel formulations but to actually increase the efficacy of the drug in clinical trials involving patients with an advanced breast cancer [38]. The increased efficacy has been proposed to be due to both increased the uptake from the intravascular space and an increased transport into cancer cells. Albumin is known to bind to endothelial glycoprotein receptor gp60, which in turn is bound to an intracellular protein cavloin-1. This process causes the formation of transcytotic vesicles known as caveolae that transport the plasma constituents within the vesicle through the endothelial cell, including the albumin-bound paclitaxel [39]. Once the drug has been delivered from the intravascular space into the intratumoral space, albumin also facilitates the intratumoral uptake of paclitaxel. A recent in vivo study showed a 33\% higher accumulation of paclitaxel in the intratumoral space for an albumin-conjugated form of paclitaxel when equal doses of paclitaxel were administered using the albumin-based and the Cremophor-formulated paclitaxel [41]. Current explanation for this phenomenon is that albumin is also able to bind to SPARC (secreted protein acid and rich in cysteine), a protein that is overexpressed in the extracellular space for a variety of cancers [39].

Abraxane (also commonly referred to as nab-paclitaxel) was approved by the FDA in 2005 for the treatment of breast cancer after the failure of the combination chemotherapy for metastatic disease or relapse within 6 months of adjuvant chemotherapy. Prior therapy should have included an anthracycline unless clinically contraindicated [42]. Several other indications are currently being pursued through aggressive clinical trials, including first-line metastatic breast cancer, nonsmall cell lung cancer, and recurrent ovarian, peritoneal, or fallopian tube cancers in platinum-sensitive patients.

2.4. Rexin-G. The cancer collagen matrix targeting nanomedicine platform is a liposome platform with a highaffinity collagen-binding motif, derived from coagulation von Willebrand factor (vWF), which is genetically engineered into liposome surface proteins [43]. The model product of this platform, Rexin-G, an Epeius proprietary product, is a replication-incompetent, pathotropic (disease-seeking) tumor matrix (collagen-) targeted retro vector encoding an $\mathrm{N}$-terminal deletion mutant of the cyclin G1 gene with potential antineoplastic activity [43,44]. The microRNA is encapsulated into the said platform. When injected intravenously, the nanoparticles $(\sim 100 \mathrm{~nm})$ seek out and accumulate in cancerous lesions wherein collagenous matrix proteins are exposed by tumor invasion, neoangiogenesis, and extracellular remodeling. The accumulation of nanoparticles within the tumor microenvironment, in the vicinity of cancer cells, enhances gene transfer efficiency via natural viral cell receptor mechanisms. In Epeius early preclinical and clinical studies, Rexin-G demonstrated a significant antitumor activity in a broad spectrum of solid tumors $[45,46]$.

Although Epeius is currently conducting a number of clinical trials in the USA and abroad for Rexin-G as therapeutic intervention for metastatic or locally advanced cancers, Rexin-G in fact has been approved for use in "all solid tumors" in the Philippines based on profound evidence of single-agent efficacy in a broad spectrum of chemoresistant tumors [43]. The clinical data including the documentation of safety and efficacy of Rexin-G from studies conducted in the Philippines has helped Rexin-G to gain Orphan Drug Designation as an effective treatment for pancreatic cancer in 2003, followed by Orphan Drug Status for both osteosarcoma and soft tissue sarcoma in 2008 [44]. By 2009, Epeius completed its Advanced Phase I/II and confirmatory Phase II trials for pancreatic cancer, sarcoma, and osteosarcoma, respectively, with all primary and secondary endpoints achieved. Consequently, Rexin-G has received FAST Track Status and priorities from the US-FDA and is in the process of applying for an accelerated approval for these clinical indications. The market in the USA may grow sharply if Phase III clinical trials go well [44].

It is worthwhile to point out that the Rexin-G technology is the first of its kind (gene therapy of cancer) accessible on the market (e.g., in the Philippines) and has been considered revolutionary in the field of cancer treatment [47].

2.5. Oncaspar. Asparaginase (ASNase), a naturally occurring enzyme expressed and produced by microorganisms, catalyzes the hydrolysis of asparagine to aspartic acid. ASNase was identified as a potential chemotherapeutic agent for acute lymphoblastic leukemia (ALL) in 1961 when it was observed that guinea pig serum-treated lymphoma-bearing mice underwent a rapid and often complete regression [48]. The potential of ASNase as a childhood ALL therapeutic was confirmed with the consecutive series of clinical trials [49].

The working mechanism behind ASNase therapeutic effect on ALL is that ASNase catalyzes the conversion of Lasparagine to aspartic acid and ammonia (as shown by the equation below) whereas leukemic cells growth depends on circulating asparagine, since that ALL leukemic cells and some other suspected tumor cells are unable to synthesize the nonessential amino acid asparagine, whereas normal cells are able to make their own asparagine [50]. The presence of ASNase deprives the leukemic cell of circulating asparagine, which leads to cell death:

$$
\text { Asparagine } \stackrel{\mathrm{km}=29 \mu \mathrm{M}}{\longrightarrow} \text { Aspartic acid }+\mathrm{NH}_{3}
$$


In the USA there are two forms of commercially available asparaginase: Elspar, an E. coli-derived asparaginase manufactured by Merck (Whitehouse Station, NJ, USA), and pegaspargase (Oncaspar), a PEGylated form of asparaginase, manufactured by Enzon Pharmaceuticals, Inc. (Bridgewater, NJ, USA) from the Merck asparaginase bulk drug product [50]. The technology of polyethylene glycol covalent conjugation to the native asparaginase increases the drug hydrodynamic radius, prolongs its circulation and retention time, decreases proteolysis, decreases renal excretion, and shields antigenic determinants from immune detection without obstructing the substrate-interaction site, and thus reduces the side effect of the native asparaginase such as hypersensitivity $[51,52]$.

Oncaspar was first approved by the US-FDA in 1994 for use in ALL patients who developed hypersensitivity to the native form of asparaginase. It was then approved by the US-FDA in 2006 as the first-line treatment of patients with ALL as a component of a multiagent thermotherapy regimen [53]. In addition to circumventing hypersensitivity to Elspar, PEGylation results in Oncaspar having a longer biological half-life than Elspar, as shown by Table 2. In most treatment protocols for ALL, Elspar is given three times weekly for six or nine doses. Because of the longer half-life of Oncaspar, it is often possible to achieve a similar or equal therapeutic effect with fewer injections [53].

This nanomedicine demonstrates again that drug surface modification (i.e., PEGylation) can significantly alter drug's pharmacokinetics and pharmacodynamics, thus eliminating some side effects (such as hypersensitivity) and/or enhancing treatment effect with a reduced dose.

2.6. Cancer Imaging Nanomedicine: Resovist and Feridex/Endorem. One of the very few fully approved inorganic-based cancer nanomedicines are superparamagnetic iron oxide (SPIO) nanoparticle agents for MR imaging. SPIO nanoparticle MR imaging contrast agents have been of great interest in research and clinical applications for the past decades [54]. While there are still some in clinical trials or experimental study stages, a few SPIO products have been approved by various regulatory bodies including the US-FDA [55]. Of these approved SPIOs, the most phenomenal products for cancer diagnostics are Resovist, Endorem, and Feridex [55].

Resovist, ferumoxide SPIO nanoparticles with $4.2 \mathrm{~nm}$ core diameters and $62 \mathrm{~nm}$ hydrodynamic diameters, is an organ-specific MRI contrast agent used for the detection and characterization of especially small focal liver lesions [56]. Resovist consists of SPIO nanoparticles coated with carboxydextran, which are accumulated by phagocytosis in cells of the reticuloendothelial system (RES) of the liver. The uptake of Resovist injection in the reticuloendothelial cells results in a decrease of the signal intensity of normal liver parenchyma on both T2- and T1-weighted images. Most malignant liver tumors do not contain RES cells and therefore do not uptake the iron particles. The resulting imaging effect is an improved contrast between the tumor (bright) and the surrounding tissue (dark). Resovist can be injected as an intravenous bolus, which allows for the immediate imaging of the liver and reduces the overall examination time.
A dynamic imaging strategy after bolus injection supports characterizing lesions. In comprehensive clinical trials, it demonstrated an excellent safety profile [57, 58]. In 2001, Resovist was approved for the European market. However, Resovist competed with Primovist, the other liver imaging agent of Bayer Schering Pharma AG. Due to this reason, the production of Resovist was unfortunately abandoned in 2009.

Endorem, marketed by Guerbet, and Feridex (approved by US-FDA in 1996), marketed by Berlex Laboratories, are also ferumoxide SPIO nanoparticles but coated with dextran instead of carboxdextran. The iron oxide core is $4.8-5.6 \mathrm{~nm}$ and the hydrodynamic diameter is about $80-150 \mathrm{~nm}$. These nanoparticles efficiently accumulate in the liver (about $80 \%$ of injected dose) and spleen (about $5-10 \%$ of injected dose) within only minutes of administration, due to RES uptake [59]. But tumor tissues such as metastases, primary liver cancer, cysts and various benign tumors, adenomas, and hyperplasia do not take these materials and thus retain their native signal intensity, so the contrast between normal and abnormal tissue is increased. Peak concentrations of iron were found in liver after 2 hours and in the spleen after 4 hours and the blood half-life is only about $6 \mathrm{~min}$ [59]. Unlike Resovist, Endorem and Feridex show some side effects such as cardiovascular problems and lumbar pain [60].

Further development of SPIO-enhanced MR imaging depends on the improved knowledge of the fundamental mechanism of SPIO agents on MR signal, the pharmacokinetic control of these agents, including appropriate selection of particle charge, size, and coating, linking these agents with molecules capable of accurate specific targeting, and the development of new imaging sequences [60].

\section{Cancer Nanomedicine in Clinical Trials}

This section is focused on the cancer nanomedicines that are in clinical trials for any cancer indications and have not been approved by FDA or other regulatory bodies for commercial production.

3.1. Therapeutic Nanomedicine in Clinical Trials. Even with development challenges, a few first-generation nanoparticle therapeutics, such as Doxil and Abraxane, have already obtained recognition in the clinical cancer research community. Considerable research and clinical trials are now being invested in qualifying nanoparticles as "platforms" for various drugs. Table 3 provides an updated summary of the nanomedicine constructs currently undergoing clinical trials as previously published by Zhang et al. [61] and Tiwari et al. [62].

A large number of preclinical nanoparticle delivery systems have been developed with potential for cancer detection and therapy. The European Science and Technology Observatory (ESTO) conducted a global survey in 2006 and identified that over 150 companies are developing nanoscale therapeutics [63]. As indicated by this blossoming research area, the synthesis and formulation possibilities for nanoparticles are almost endless considering the ability to incorporate various chemical and biology entities that provide both imaging and therapeutic capabilities. However, this multimodality 
approach combined with the ability to control and target the delivery of a therapeutic agent requires a sophisticated engineering of nanoparticles. For example, multiple factors affect the pharmacokinetic behavior of nanoparticles, but the surface charge, size, nanoparticle shape, and stealth properties are among the most critical. Researchers have exploited these properties at the benchtop for introduction into various preclinical models for the optimization of the stability and delivery characteristics of the nanoparticle. More significantly, this research work has now also led into the introduction of nanoparticle candidates into the clinical and commercial market.

In particular, therapeutically loaded nanomedicines have the potential for achieving improved therapeutic indexes for cancer treatment. Nanoparticles have shown a significant promise as improved drug delivery systems improving the formulation characteristics of the existing drugs that may have poor administration. Several commercial examples now exist, confirming the promise of nanoparticle reformulation approaches to reduce drug side effects, while potentially increasing efficacy. One additional promise of nanoparticles is the reformulation of therapeutic agents that have failed in clinical development due to pharmacology challenges. For example, Shim et al. [64] recently described a proof of a principle nanoparticle approach to overcome drug delivery challenges and revive the clinical potential of wortmannin.

A series of clinical studies have substantiated the potential of nanoparticle-based therapeutics for demonstrating the enhanced tumor accumulation of delivered therapeutics with less adverse effects. Although most commercialized nanomedicines have been based on a passive-targeting strategy that exploits the EPR effect, there is an increasing need for the development of active-targeting technologies. The leading strategy has been the conjugation of ligand molecules that specifically bind to receptors that are overexpressed on tumor cells relative to normal cells [62].

As indicated during previous discussions and by a recent review [65], six common types of nanoparticles are approved or in late stages of clinical trials: polymer-drug conjugates, micelles, protein-based carriers, liposomes, polymeric nanoparticles, and inorganic nanoparticles. As previously discussed and seen from Table 3 a majority of nanoparticles under clinical/commercial development are therapeutics, and most of them are organic based due to a relatively low toxicity and high biocompatibility.

To further extend the capabilities of nanoparticles for simultaneous imaging and therapeutic applications, researchers have begun to increasingly exploit various inorganic matrices, such as gold, iron oxide, quantum dots, and silica nanoparticles [66]. In this respect, nanomedicines can be developed as theranostics, which possess both imaging and therapy capabilities.

3.2. Theranostics in Clinical Trials. Cancer nanotheranostics are the use of nanoparticles for combined diagnosis and therapy in cancer applications. Incorporating imaging capability into a nanoparticle is a very useful attribute for monitoring the distribution of the treatment in vivo. This provides direct and confirmatory information about the mechanism of action for a specific patient, thereby providing a significant advantage over more traditional drug therapies for cancer treatment. The development of multifunctional nanoparticles has the potential for the rapid disease verification and identification of the effected tissues for improved treatment protocols. Wang et al. [66] indicated in a recent review article that a combined technique will result in an acceleration of the drug development and improved disease management with reduced risks and costs.

The clinical development of theranostic agents is still preliminary and several challenges, such as efficient and targeted guidance of the therapeutic/imaging nanoparticles, still exist [67]. In addition, the potency of the nanoparticle could be further improved with multimodality treatment options. However, one nanoparticle platform which is progressing from the benchtop to the clinical bedside is gold nanoparticles. Their inherit properties make them appealing as the material can be easily manufactured and functionalized with both imaging/therapeutic agents and targeting vectors [68]. Initial clinical studies have been completed with gold nanoparticles coated with citrate and thiolated PEG/tumor necrosis factor- $\alpha$ (TNF- $\alpha)[69,70]$. The dose limiting toxicity of TNF- $\alpha$ was significantly improved with the developed gold nanoparticle formulation.

\section{Summary and Perspectives}

The biggest challenge that nanomedicine faces at present is meeting all the safety guidelines required for gaining clinical acceptance, particularly those required by the FDA and other regulatory bodies. Over the past decade, various nanoparticle/nanomedicine platforms have been screened and studied in terms of their size, shape, and surface properties to meet these guidelines and market request. These include (1) "target-specificity": ideally, a nanomedicine will accumulate mainly in the diseased organs/sites, bypassing normal ones, (2) a proper size (often ultrasmall): thus they can be eliminated from the body preferably through the renal filtration system, and (3) nontoxic and biocompatible, with a surface made up of natural polymers/biomolecules. Versatility of a nanomedicine is also highly desirable, which means that multifunctional therapeutic agents and multimodality imaging capacity (i.e., optical, MRI, SPECT, PET, and/or CT) can be combined into one nanomedicine that not only will facilitate the early diagnosis of diseases, but will also have the potential to monitor in real time the progress of the therapeutic delivery.

In spite of all the challenges, nanotechnology has become closely related with cancer care today and has been applied in an evolutionary manner to improve the properties of cancer therapeutic, diagnostics, and other health care products. Biotech, pharmaceutical, and medical sciences companies have been active parts of the evolution and are dynamic collaborators with researchers, government, and educational institutions in developing and translating cancer nanomedicines. Based on the full spectrum of cancer nanomedicines in clinical trials and on the market that have been discussed in this paper, it is highly expected that the forthcoming generations of nanomedicines will 
have targeting moiety, may carry multiple drugs that could potentially be released in a controlled manner, and will be equipped with an imaging capacity.

\section{Conflict of Interests}

The authors claim no financial interests with any of the companies that are mentioned in the paper.

\section{References}

[1] National Science and Technology Council Committee on Technology, The National Nanotechnology Initiative: Research and Development Leading to a Revolution in Technology and Industry, Office of Sciences and Technology Policy, Washington, DC, USA, 2005.

[2] "Drug delivery," in The Handbook of Experimental Pharmacology, M. Schafer-Korting, Ed., pp. 55-86, Springer, Berlin, Germany, 2010.

[3] S. E. McNeil, "Nanoparticle therapeutics: a personal perspective," Wiley Interdisciplinary Reviews, vol. 1, no. 3, pp. 264-271, 2009.

[4] S. McNeil, "Nanoparticle therapeutics: a personal perspective," in Proceedings of the MRS Functionalized Nanobiomaterials for Medical Applications Workshop, Denver, Colo, USA, 2010.

[5] W. E. Bawarski, E. Chidlowsky, D. J. Bharali, and S. A. Mousa, "Emerging nanopharmaceuticals," Nanomedicine, vol. 4, no. 4, pp. 273-282, 2008.

[6] H. S. Choi and J. V. Frangioni, "Nanoparticles for biomedical imaging: fundamentals of clinical translation," Molecular Imaging, vol. 9, no. 6, pp. 291-310, 2010.

[7] M. L. Immordino, F. Dosio, and L. Cattel, "Stealth liposomes: review of the basic science, rationale, and clinical applications, existing and potential," International Journal of Nanomedicine, vol. 1, no. 3, pp. 297-315, 2006.

[8] M. R. Green, G. M. Manikhas, S. Orlov et al., "Abraxane, a novel Cremophor-free, albumin-bound particle form of paclitaxel for the treatment of advanced non-small-cell lung cancer," Annals of Oncology, vol. 17, no. 8, pp. 1263-1268, 2006.

[9] E. H. Chang, "Nanomedicines: improving current cancer therapies and and diagnosis," Nanomedicine, vol. 3, no. 4, p. 339, 2007.

[10] J. M. Stern, J. Stanfield, W. Kabbani, J. T. Hsieh, and J. A. Cadeddu, "Selective prostate cancer thermal ablation with laser activated gold nanoshells," Journal of Urology, vol. 179, no. 2, pp. 748-753, 2008.

[11] Y. Barenholz, "Doxil—the first fda-approved nano-drug: lessons learned," Journal of Control Release, vol. 160, no. 2, pp. 117-134, 2012.

[12] A. A. Gabizon, "Pegylated liposomal doxorubicin: metamorphosis of an old drug into a new form of chemotherapy," Cancer Investigation, vol. 19, no. 4, pp. 424-436, 2001.

[13] A. Gabizon, H. Shmeeda, and Y. Barenholz, "Pharmacokinetics of pegylated liposomal doxorubicin: review of animal and human studies," Clinical Pharmacokinetics, vol. 42, no. 5, pp. 419-436, 2003.

[14] B. Čeh, M. Winterhalter, P. M. Frederik, J. J. Vallner, and D. D. Lasic, "Stealth liposomes: from theory to product," Advanced Drug Delivery Reviews, vol. 24, no. 2-3, pp. 165-177, 1997.

[15] A. Gabizon, R. Catane, B. Uziely et al., "Prolonged circulation time and enhanced accumulation in malignant exudates of doxorubicin encapsulated in polyethylene-glycol coated liposomes," Cancer Research, vol. 54, no. 4, pp. 987-992, 1994.

[16] Y. Malam, M. Loizidou, and A. M. Seifalian, "Liposomes and nanoparticles: nanosized vehicles for drug delivery in cancer," Trends in Pharmacological Sciences, vol. 30, no. 11, pp. 592-599, 2009.

[17] F. M. Muggia, J. D. Hainsworth, S. Jeffers et al., "Phase II study of liposomal doxorubicin in refractory ovarian cancer: antitumor activity and toxicity modification by liposomal encapsulation," Journal of Clinical Oncology, vol. 15, no. 3, pp. 987-993, 1997.

[18] A. Gabizon, D. Goren, A. T. Horowitz, D. Tzemach, A. Lossos, and T. Siegal, "Long-circulating liposomes for drug delivery in cancer therapy: a review of biodistribution studies in tumorbearing animals," Advanced Drug Delivery Reviews, vol. 24, no. 2-3, pp. 337-344, 1997.

[19] D. N. Waterhouse, P. G. Tardi, L. D. Mayer, and M. B. Bally, "A comparison of liposomal formulations of doxorubicin with Drug Administered in free form: changing toxicity profiles," Drug Safety, vol. 24, no. 12, pp. 903-920, 2001.

[20] T. Lammers, W. E. Hennink, and G. Storm, “Tumour-targeted nanomedicines: principles and practice," British Journal of Cancer, vol. 99, no. 3, pp. 392-397, 2008.

[21] L. Harris, G. Batist, R. Belt et al., "Liposome-encapsulated doxorubicin compared with conventional doxorubicin in a randomized multicenter trial as first-line therapy of metastatic breast carcinoma," Cancer, vol. 94, no. 1, pp. 25-36, 2002.

[22] D. S. Alberts, F. M. Muggia, J. Carmichael et al., "Efficacy and safety of liposomal anthracyclines in Phase I/II clinical trials," Seminars in Oncology, vol. 31, no. 13, pp. 53-90, 2004.

[23] Sopherion Therapeutics, "Myocet: doxorubicin hydrochloride (Liposome) for injection," 2001, http://www.sopherion.com/ pdf/PIEnglishv0-2.pdf.

[24] E. A. Forssen, "The design and development of DaunoXome for solid tumor targeting in vivo," Advanced Drug Delivery Reviews, vol. 24, no. 2-3, pp. 133-150, 1997.

[25] A. Fassas and A. Anagnostopoulos, "The use of liposomal daunorubicin (DaunoXome) in acute myeloid leukemia," Leukemia and Lymphoma, vol. 46, no. 6, pp. 795-802, 2005.

[26] J. A. Sparano and E. P. Winer, "Liposomal anthracyclines for breast cancer," Seminars in Oncology, vol. 28, no. 4, pp. 32-40, 2001.

[27] E. A. Forssen and M. E. Ross, "DaunoXome treatment of solid tumors preclinical and clinical investigations," Journal of Liposome Research, vol. 4, no. 1, pp. 481-512, 1994.

[28] T. M. Allen and F. J. Martin, "Advantages of liposomal delivery systems for anthracyclines," Seminars in Oncology, vol. 31, supplement 13, pp. 5-15, 2004.

[29] Galen US, "DaunoXome: daunorubicin citrate liposome injection," 2011, http://daunoxome.com/downloads/DaunoXome\%20PI.pdf.

[30] Clinicaltrials.gov, U.S. National Institutes of Health, Search for "DaunoXome" AND "Phase 3", http://clinicaltrials.gov/.

[31] H. I. Chang, M. Y. Cheng, and M. K. Yeh, "Clinically-proven liposome-based drug delivery: formulation, characterization and therapeutic efficacy," vol. 1, Article ID 195, 2012.

[32] S. Dromi, V. Frenkel, A. Luk et al., "Pulsed-high intensity focused ultrasound and low temperature-sensitive liposomes for enhanced targeted drug delivery and antitumor effect," Clinical Cancer Research, vol. 13, no. 9, pp. 2722-2727, 2007.

[33] R. T. P. Poon and N. Borys, "Lyso-thermosensitive liposomal doxorubicin: a novel approach to enhance efficacy of thermal 
ablation of liver cancer," Expert Opinion on Pharmacotherapy, vol. 10, no. 2, pp. 333-343, 2009.

[34] Clinicaltrials.gov, U.S. National Institutes of Health, Search for "ThermoDo" AND "Phase 3", http://clinicaltrials.gov/.

[35] "Celsion Progresses with ThermoDox," 2012, http://finance .yahoo.com/news/celsion-progresses-thermodox-211903842 .html.

[36] “Clinicaltrials.gov," U.S. National Institutes of Health, Search for "ThermoDox", http://clinicaltrials.gov/.

[37] P. B. Schiff and S. B. Horwitz, "Taxol stabilizes microtubules in mouse fibroblast cells," Proceedings of the National Academy of Sciences of the United States of America, vol. 77, no. 3, pp. 1561-1565, 1980.

[38] V. Guarneri, M. V. Dieci, and P. Conte, "Enhancing intracellular taxane delivery: current role and perspectives of nanoparticle albumin-bound paclitaxel in the treatment of advanced breast cancer," Expert Opinion in Pharmacotherapy, vol. 13, no. 3, pp. 395-406, 2012.

[39] M. J. Hawkins, P. Soon-Shiong, and N. Desai, "Protein nanoparticles as drug carriers in clinical medicine," Advanced Drug Delivery Reviews, vol. 60, no. 8, pp. 876-885, 2008.

[40] F. Kratz, "Albumin as a drug carrier: design of prodrugs, drug conjugates and nanoparticles," Journal of Controlled Release, vol. 132, no. 3, pp. 171-183, 2008.

[41] N. Desai, V. Trieu, Z. Yao et al., "Increased antitumor activity, intratumor paclitaxel concentrations, and endothelial cell transport of cremophor-free, albumin-bound paclitaxel, ABI007, compared with cremophor-based paclitaxel," Clinical Cancer Research, vol. 12, no. 4, pp. 1317-1324, 2006.

[42] Abraxis BioScience LLC, "ABRAXANE Healthcare Professional Prescribing Information," http://www.abraxane.com/ hcp/download/Abraxane_Prescribing_Information.pdf.

[43] Epeius Biotech, http://www.epeiusbiotech.com/oncologyRexinG-factsheet.asp.

[44] E. M. Gordon and F. L. Hall, "Rexin-G, a targeted genetic medicine for cancer," Expert Opinion on Biological Therapy, vol. 10, no. 5, pp. 819-832, 2010.

[45] S. P. Chawla, V. S. Chua, L. Fernandez et al., "Phase I/II and phase II studies of targeted gene delivery in vivo: intravenous Rexin-G for chemotherapy-resistant sarcoma and osteosarcoma," Molecular Therapy, vol. 17, no. 9, pp. 1651-1657, 2009.

[46] S. P. Chawla, V. S. Chua, L. Fernandez et al., "Advanced phase I/II studies of targeted gene delivery in vivo: intravenous rexin-G for gemcitabine-resistant metastatic pancreatic cancer," Molecular Therapy, vol. 18, no. 2, pp. 435-441, 2010.

[47] E. Galanis, S. K. Carlson, N. R. Foster et al., "Phase I trial of a pathotropic retroviral vector expressing a cytocidal cyclin G1 construct (Rexin-G) in patients with advanced pancreatic cancer," Molecular Therapy, vol. 16, no. 5, pp. 979-984, 2008.

[48] J. D. Broome, "Evidence that the L-asparaginase activity of guinea pig serum is responsible for its antilymphoma effects," Nature, vol. 191, no. 4793, pp. 1114-1115, 1961.

[49] L. Tallal, C. Tan, H. Oettgen et al., "E. coli L-asparaginase in the treatment of leukemia and solid tumors in 131 childrens," Cancer, vol. 25, no. 2, pp. 306-320, 1970.

[50] V. I. Avramis and P. N. Tiwari, "Asparaginase (native ASNase or pegylated ASNase) in the treatment of acute lymphoblastic leukemia," International journal of nanomedicine, vol. 1, no. 3 , pp. 241-254, 2006.

[51] P. S. Gaynon, "Childhood acute lymphoblastic leukaemia and relapse," British Journal of Hematology, vol. 131, no. 5, pp. 579-587, 2005.
[52] M. Jarrar, P. S. Gaynon, A. P. Periclou et al., "Asparagine depletion after pegylated E. coli asparaginase treatment and induction outcome in children with acute lymphoblastic leukemia infirst bone marrow relapse: a Children's Oncology Group Study (CCG-1941)," Pediatric Blood and Cancer, vol. 47, no. 2, pp. 141-146, 2006.

[53] H. J. Lenz, "Management and preparedness for infusion and hypersensitivity reactions," Oncologist, vol. 12, no. 5, pp. 601-609, 2007.

[54] P. Reimer and B. Tombach, "Hepatic MRI with SPIO: detection and characterization of focal liver lesions," European Radiology, vol. 8, no. 7, pp. 1198-1204, 1998.

[55] Y. X. J. Wang, S. M. Hussain, and G. P. Krestin, "Superparamagnetic iron oxide contrast agents: physicochemical characteristics and applications in MR imaging," European Radiology, vol. 11, no. 11, pp. 2319-2331, 2001.

[56] P. Reimer and T. Balzer, "Ferucarbotran (Resovist): a new clinically approved RES-specific contrast agent for contrastenhanced MRI of the liver: properties, clinical development, and applications," European Radiology, vol. 13, no. 6, pp. 1266-1276, 2003.

[57] B. Hamm, T. Staks, M. Taupitz et al., "Contrast-enhanced MR imaging of liver and spleen: first experience in humans with a new superparamagnetic iron oxide," Journal of Magnetic Resonance Imaging, vol. 4, no. 5, pp. 659-668, 1994.

[58] B. Hamm, T. Staks, and M. Taupitz, "A new superparamagnetic iron oxide contrast agent for magnetic resonance imaging," Investigative Radiology, vol. 29, no. 2, pp. S87-S89, 1994.

[59] R. Weissleder, D. D. Stark, B. L. Engelstad et al., "Superparamagnetic iron oxide: pharmacokinetics and toxicity," American Journal of Roentgenology, vol. 152, no. 1, pp. 167-173, 1989.

[60] Y. X. Wang, "Superparamagnetic iron oxide based MRI contrast agents: current status of clinical application," Quantitative Imaging in Medicine and Surgery, vol. 1, no. 1, pp. 35-40, 2011.

[61] L. Zhang, F. X. Gu, J. M. Chan, A. Z. Wang, R. S. Langer, and O. C. Farokhzad, "Nanoparticles in medicine: therapeutic applications and developments," Clinical Pharmacology and Therapeutics, vol. 83, no. 5, pp. 761-769, 2008.

[62] M. Tiwari, "Nano cancer therapy strategies," Journal of Cancer Research and Therapeutics, vol. 8, no. 1, pp. 19-22, 2012.

[63] S. . Karve, M. E. Werner, R. Sukumar et al., "Revival of the abandoned therapeutic wortmannin by nanoparticle drug delivery," Proceedings of the National Academy of Sciences of the United States of America, vol. 109, no. 21, pp. 8230-8235, 2012.

[64] G. Shim, S. Lee, H. Choi et al., "Nanomedicines for receptormediated tumor targeting," Recent Patents on Nanomedicine, vol. 1, no. 2, pp. 138-148, 2012.

[65] A. Naveed, H. Fessi, and A. Elaissari, "Theranostic applications of nanoparticles in cancer," Drug Discovery Today, vol. 17, no. 17-18, pp. 928-934, 2012.

[66] A. Z. . Wang, R. Langer, and O. C. Farokhzad, "Nanoparticle delivery of cancer drugs," Annual Review of Medicine, vol. 63, pp. 185-198, 2012.

[67] M. P. Melancn, R. J. Stafford, and C. Li, "Challenges to effective cancer nanotheranostics," Journal of Controlled Release, vol. 164, pp. 177-182, 2012.

[68] N. Trivedi, N. Patel, U. M. Upadhyay, and S. Sha, "Gold nanoparticulate drug delivery system: a review," Pharmacie Globale International Journal of Comphrehesive Pharmacy, vol. 3, no. 6, pp. 1-5, 2012. 
[69] S. K. Libutti, G. F. Paciotti, L. Myer, R. Haynes, W. Gannon, and M. Walker, "Results of a completed phase I clinical trial of CYT6091: a peglated colloidal gold-TNF," Nanomedicine, vol. 27, no. 15, p. 3586, 2009.

[70] S. K. Libutti, G. F. Paciotti, A. A. Byrnes et al., "Phase I and pharmacokinetic studies of CYT-6091, a novel PEGylated colloidal gold-rhTNF nanomedicine," Clinical Cancer Research, vol. 16, no. 24, pp. 6139-6149, 2010. 

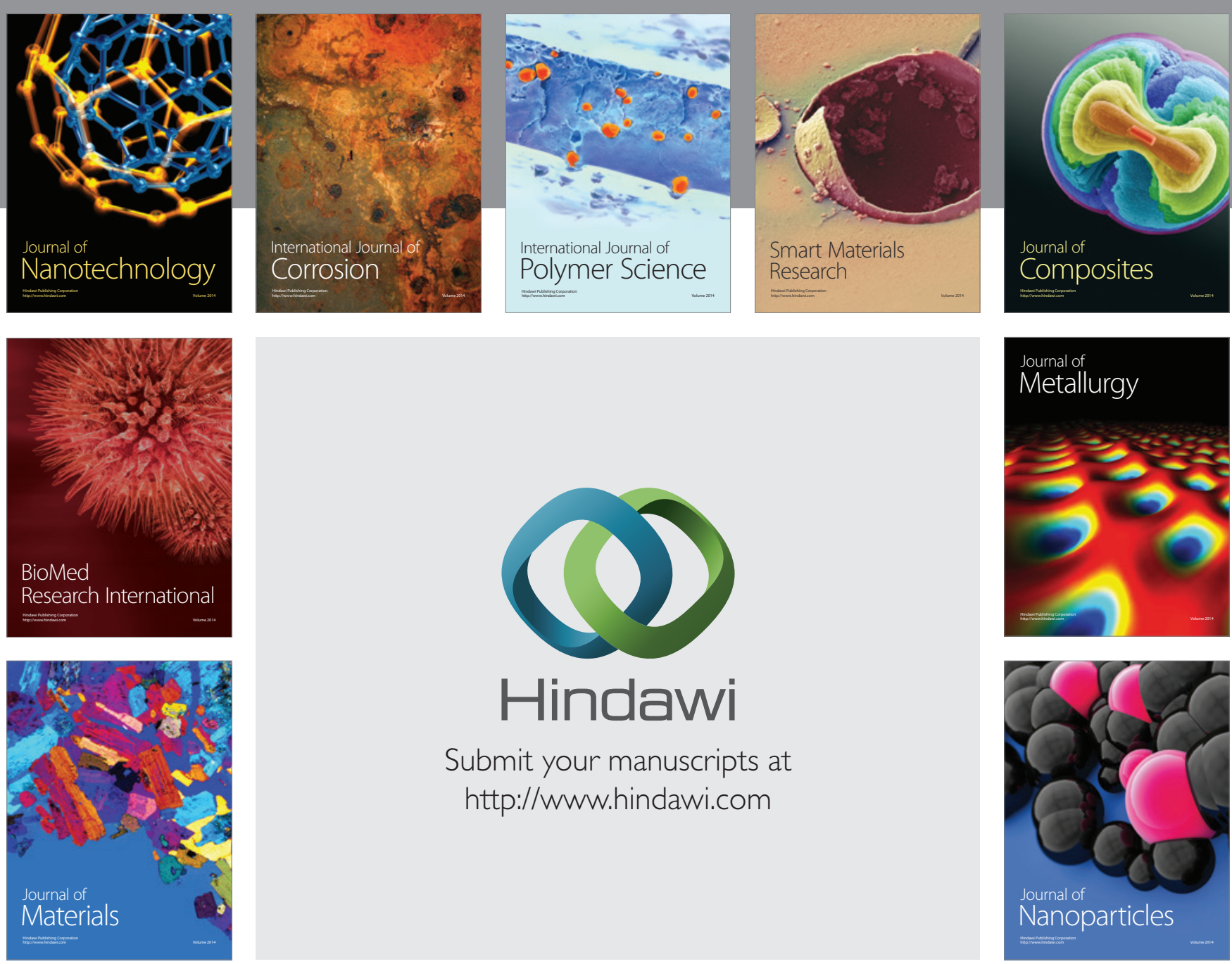

Submit your manuscripts at http://www.hindawi.com
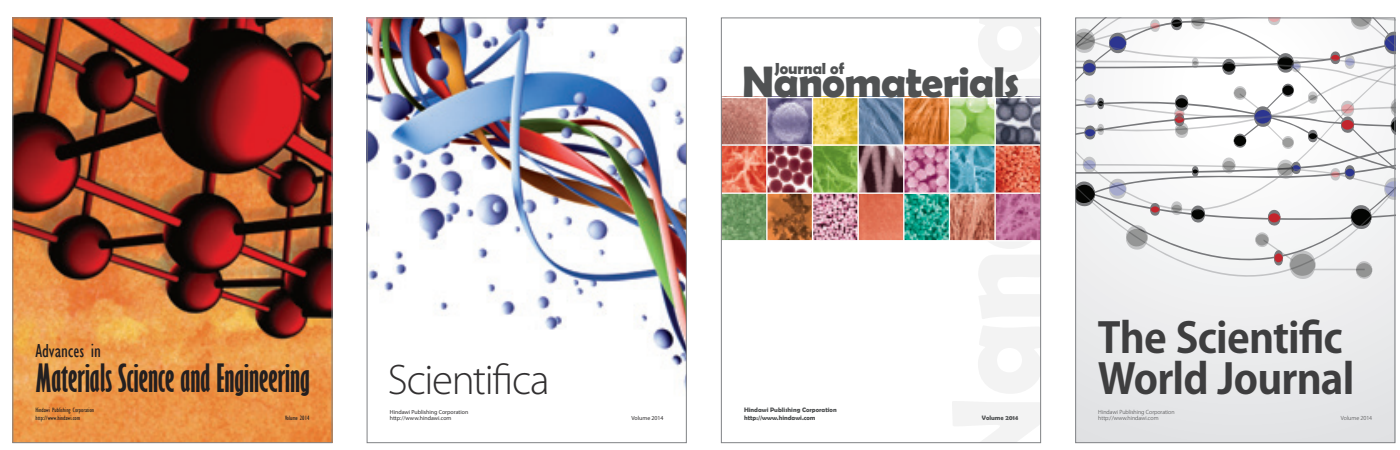

\section{The Scientific World Journal}
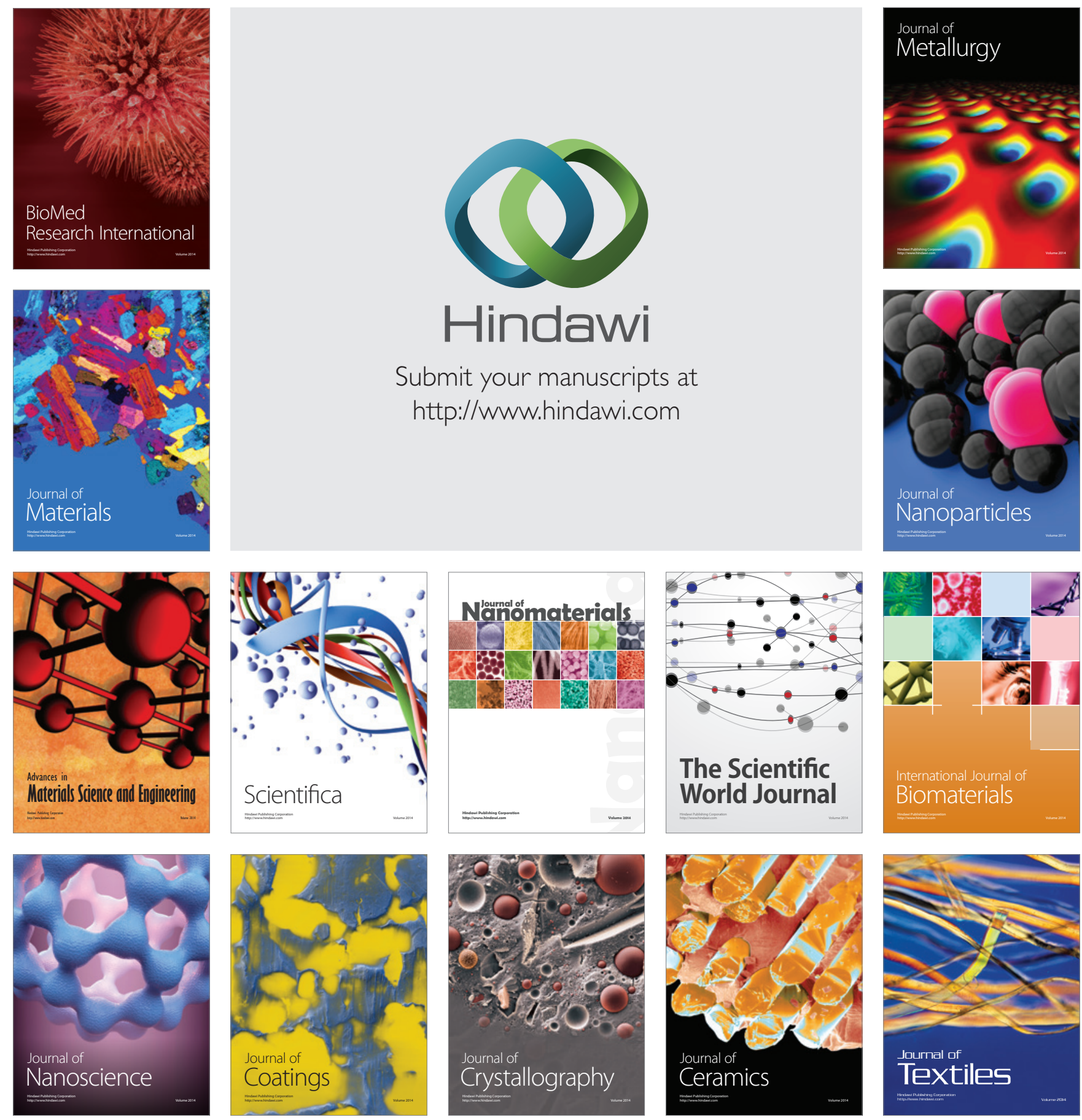